\title{
Mixed Reality for Virtual Jewellery Fitting
}

\author{
Vincent W. S. Cheng \\ The University of Hong Kong
}

\author{
S. H. Choi \\ The University of Hong Kong \\ H. H. Cheung \\ The University of Hong Kong
}

The Hong Kong jewellery industry is facing challenges in adopting e-commerce, especially for product visualisation, personalised marketing, and building customer faith. To tackle these challenges, a mixed reality for virtual jewellery fitting (MRVJF) system is developed for jewellery retailing. The MRVJF is designed and implemented in consideration of time and budget limitations, availability of functionalities, and potential benefits. It incorporates mixed reality, face recognition, and hand recognition technologies to improve jewellery visualisation and user-friendliness with interactive virtual fitting on smartphones. The MRVJF is aimed to increase the competitiveness of the Hong Kong jewellery retailers in the global commodity chain.

Keywords: Mixed Reality, Virtual Fitting, Facial Recognition, Jewellery

\section{INTRODUCTION}

The jewellery industry in Hong Kong is recognised as a leading jade production and pearl trading centre, and is known for its flexibility in accommodating customer needs (Hong Kong Trade Development Council, 2018). However, it faces various challenges, particularly the inventory cost of maintaining product visibility for improvement of customer shopping experiences. First, unclear visualisation of tailor-made jewellery designs leads to high defective cost and customer dissatisfaction. Second, high inventory cost is required to showcase all designs in retail stores. Third, some online shopping platforms lack effective tools for product visualisation and customer interactions.

To tackle the above challenges and promote e-commerce in the jewellery industry, virtual jewellery fittings have been implemented to enhance customer shopping experiences (Rodriguez et al., 2014). The existing virtual jewellery fittings commonly utilise static image fitting with limited interaction between customers and products. Other virtual jewellery fittings utilise quick response (QR) code markers to track the customer position. However, the manipulation of QR code causes the fitting experience being unrealistic and not user-friendly. 
To improve the fitting experiences, this paper proposes a virtual jewellery fitting system that incorporates mixed reality (MR) with face and hand recognition to track the customer position in realtime without the aid of markers. It simplifies the virtual fitting conditions and procedures.

MR refers to the incorporation and anchoring of the virtual computer graphics objects into a real three-dimensional environment, allowing the virtual objects to interact with the real environment in realtime. MR is an on-trend channel to attract new customers due to the emerging applications like Snapchat, Pokémon Go, and Vive.

\section{RESEARCH OBJECTIVES, SIGNIFICANCE, AND ORIGINALITY}

\section{Objectives}

In this paper, a mixed reality for virtual jewellery fitting (MRVJF) system is developed for customers to experience interactive virtual jewellery fitting anytime anywhere. The objectives are listed as follows:

1. To tackle challenges in the Hong Kong jewellery retail industry;

2. To increase jewellery business sales, profit margin, and reduce inventory cost;

3. To enhance customer shopping experiences, services, and satisfaction;

4. To enhance virtual jewellery visualisation including markerless tracking, positional accuracy, and jewellery realism;

5. To promote marketing with unique selling points through social media.

\section{Significance}

The significance of the MRVJF is to increase the competitiveness of the Hong Kong jewellery retailers in the global commodity chain through technologies. The MRVJF integrates brick-and-motor retail stores with e-commerce, allowing customers to virtually fit and purchase jewellery online with confidence. The jewellery visualisation improvement reduces tailor-made jewellery defective rate, transforms customer shopping habits, and broadens the applications of virtual jewellery fitting from entertainments to facilitate business operations.

\section{Originality}

The originality of the MRVJF is summarised as follows:

1. Available literature shows that the proposed markerless and interactive virtual jewellery fitting for smartphones is the pioneer application available for customers;

2. Markerless face and hand recognition position tracking modules are utilised to improve customer experiences;

3. The original photo shooting requirements are proposed to improve jewellery realisation and reduce the MRVJF development cost;

4. A positional fine-tuning algorithm is developed to enhance the positional accuracy of virtual jewellery products.

\section{LITERATURE REVIEW}

\section{Virtual Fitting}

Virtual jewellery fitting overlays virtual products on customer images to visualise the fitting effect. Mobile virtual jewellery fittings had three limitations (Best Photo Apps, 2018; Infotech, 2018; Optimumbrew Technology, 2017). First, customers were required to manually drag the jewellery to the preferred position on a pre-taken image. This repetitive dragging action was time consuming and inconvenient. Second, the static jewellery fitting image lacked dynamic and real-time interaction between the customer and the jewellery which diminished customer shopping experiences. Last, the virtual jewellery appeared artificial and being separated from customers due to unfit necklace shape and artificial computer aided design rendering. 
CaratLane (2018) improved the above limitations by developing a dynamic virtual jewellery fitting. Customers pre-recorded a video for virtual earring fitting which partly tackled the first two limitations. However, real-time interaction, ring and necklace fitting were still unavailable. Furthermore, the fixed posture in the video limited the jewellery interaction and the viewing angle.

To enable real-time interactive virtual fitting, researches on mixed reality virtual fitting were conducted. Interactive MR virtual jewellery fitting was developed utilising markers to position virtual objects (Radhika et al., 2016; Yang et al., 2014). However, marker detection was not user-friendly due to the complexity of marker manipulation. Furthermore, the marker's white edges diminished the realism effect of the virtual fitting.

Zhang et al. (2017) developed a markerless virtual eyeglasses fitting to assist the opticians in determining the effects of reflection and refraction of the lens. However, the initial set up took five minutes to manually position the eyeglasses model onto the customer. The long initial set up time diminished the customer fitting experience.

Choi et al. (2015) proposed an radio frequency identification based system that integrated smart mirrors for virtual fitting of apparel products. The system could automatically recommend matching products to enhance customer shopping experiences.

Furthermore, Chow Sang Sang, a famous jewellery chain store in Hong Kong, partnered with Mirum in developing an MR smart mirror for virtual necklace fitting (Intel, 2016). An Intel RealSense 3D camera was utilised for face recognition with the ability to stabilise long distance detection on customer interaction. However, a few problems remain to be addressed: (1) low display and camera resolution of roughly 640 x 480 pixels; (2) inaccurate necklace positioning; (3) inability to support multipeople jewellery fitting, earring, and ring fitting; (4) a lack of mobility of the virtual fitting platform.

\section{Research Gap in Virtual Jewellery Fitting}

Indeed, real-time interactive mobile virtual jewellery fitting is unpopular due to the following reasons. First, high development costs are required to utilise advanced technology. Second, vendors lack experience in the MR virtual fitting development, causing hesitation to be the first-mover. Third, MR development platforms were unavailable until their release in 2017. Fourth, MR was not implemented for business purposes due to unrealistic virtual fitting effects caused by artificial jewellery models and inaccurate jewellery positions. Last, there is a lack of demand in virtual jewellery fitting due to unpopularity and unawareness of its application.

A real-time interactive virtual jewellery fitting smart mirror was developed (Intel, 2016). However, the smart mirror must be used in retail stores which limits the advantages of virtual fitting for ecommerce. Migrating the smart mirror application into a mobile application will enhance the ubiquity of virtual fitting. Although the smart mirror application codes and mechanisms are similar to mobile applications, the camera and computing power differences limit the development of mobile virtual fitting. First, unlike the selected three-dimensional (3D) camera in the smart mirror, face recognition must be implemented on different two-dimensional (2D) front-facing smartphone cameras. The camera quality, variety of camera models and 2D limitation are challenges for mobile face recognition. Second, the application and computing algorithms must be simplified to run smoothly across different smartphones due to limited computing power in smartphones.

In view of the research gap above, this paper therefore proposes to develop a virtual jewellery fitting system that incorporates mixed reality on mobile devices to address the following main issues: (1) the ease-of-use, mobility and ubiquity of virtual fitting; (2) the accuracy and speed of positional tracking of virtual jewellery; (3) the realism effect of virtual fitting; and (4) the time and budget constraints, as well as the investment concerns of jewellery retailers. 


\section{METHODOLOGY}

\section{Mixed Reality for Virtual Jewellery Fitting}

The proposed MRVJF provides customers with an interactive and markerless virtual jewellery fitting experience to visualise the jewellery matching effect on their smartphone. The mechanism of the MRVJF is to first track the customer positions with the real-time face and hand recognition systems. Second, jewellery products with transparent background are overlaid onto the tracked customer positions. Third, customers review different jewellery products and the matching effect. Last, makeup, multiple background simulations and value-added features are provided to enhance the beauty and immersive experience.

Beauty, user-friendliness, and cost are the essential requirements in the MRVJF development. Possible issues are summarised below.

1. The jewellery model should reflect the genuine outlook of the jewellery. Jewellery realism effect is optimised by improving the computer aided design rendering, jewellery photo quality, jewellery positioning angle and the background removal technique;

2. Jewellery should switch smoothly for customers to browse, match, and compare;

3. The overlaid jewellery positions may not match the targeted positions due to the lack of specific facial landmarks in the ear and neck position. The jewellery positions are only overlaid according to the overall facial landmarks.

\section{Application Development Platform}

Lens Studio is an MR lens development platform created by Snapchat aiming for users to apply funny filters on photos and share them in the Snapchat community. This platform integrates a hand recognition system and an accurate face recognition system with multiple face recognition algorithm based on the active shape model. Objects and templates including camera, light, sprite, and head binding are available for developers, facilitating Lens Studio to be a user-friendly development platform.

Utilising Lens Studio to develop the MRVJF offers the below advantages. First, less effort is required for attracting customers to install an additional application. As Snapchat is a popular social media platform with 186 million daily users (Statista, 2018), Snapchat users can experience the jewellery fitting through Snapchat without downloading an additional application. Second, Snapchat users are familiar with the MR controls, hence less guidance is required. Third, Snapchat facilitates the promotion of the MRVJF. Customers can share their fitting experiences directly in Snapchat with their friends. Fourth, Lens Studio comprises built-in face and hand recognition modules, computer libraries and support iOS and Android platform. Face and hand recognition algorithms are no longer required to be constructed by the developers. Last, developers are not required to build two applications for the iOS and the Android platform respectively due to the difference in mobile systems.

On the other hand, developing the MRVJF through Lens Studio faces three disadvantages. First, the MRVJF must be run through Snapchat. Customers without Snapchat would need to install and register in Snapchat. Second, Snapchat lacks the function to record the jewellery view history of customers. The data of viewed jewellery products cannot be utilised to identify customer preferences. Third, jewellery cannot be purchased directly in the MRVJF due to the lack of connection with the online shopping platform. Despite these disadvantages, Lens Studio is still adopted as it can instantiate the features of virtual jewellery fitting and bottlenecks of the MRVJF, particularly on positional tracking and realism effect.

\section{The Active Shape Model}

Lens Studio implements the active shape model (ASM) algorithm in its face recognition system. The ASM is a face tracking algorithm formed by iteratively detecting and grouping the landmarks from sample images (Cootes et al., 1995). The ASM consists of eight main steps, including 1) Train Sample; 2) Develop a normalised model; 3) Transform RGB colour model to YCbCr colour model; 4) Build the mean model; 5) Match mean model to object; 6) Search for a better match; 7) Update match data; and 8) Target object identified. 
For face detection, landmarks are set up on the featured points and shape boundaries of the face such as face boundary, eyeball, and mouth (Lee et al., 2015). Adjacent landmarks are grouped into shapes which are represented as vectors. By training the ASM with pre-marked faces, a mean shape is formed by repeatedly rotating, scaling, and translating each shape until the resulting shape is normalised. The mean shape is then iteratively used to match with the image by searching the image for better placement of the mean shape and update its location information (Milborrow \& Nicolls, 2008; Setyati et al., 2013). The ASM enables markerless real-time face tracking on the 2D camera in smartphones.

\section{THE MIXED REALITY FOR VIRTUAL JEWELLERY FITTING SYSTEM}

\section{Basic Functions of the MRVJF}

Virtual Jewellery Model

Jewellery models are essential to display jewellery in the MRVJF. 2D images and 3D models can be input into Lens Studio. 2D images are photos taken from real jewellery products to capture their authentic look. A Portable Network Graphics (PNG) format front-facing jewellery image with a transparent background is input to Lens Studio. To reduce the application size and enhance the frames per second, the maximum image resolution supported by Lens Studio is 2048 x 2048 pixels.

\section{Face Recognition Module}

After preparing the jewellery model, the face recognition module - head binding is set up in Lens Studio. The head binding provides face tracking functions and overlays virtual jewellery onto the customer's face. In figure 1, the blue mesh is the face occluder in the shape of a human head. The occluder's shape can be replaced by a 3D scanned head model to improve the occluding effect. The occluder occludes the virtual jewellery when jewellery is behind the customer's face. It occludes both 3D meshes and $2 \mathrm{D}$ images by calculating the $3 \mathrm{D}$ positions of the $2 \mathrm{D}$ images. In figure 1 , the left earring is occluded by the occluder. This effect is necessary as it would be abnormal if the earring which is occluded by the face wrongly appears onto the face.

FIGURE 1

\section{LAYOUT OF THE FACE RECOGNITION MODULE}

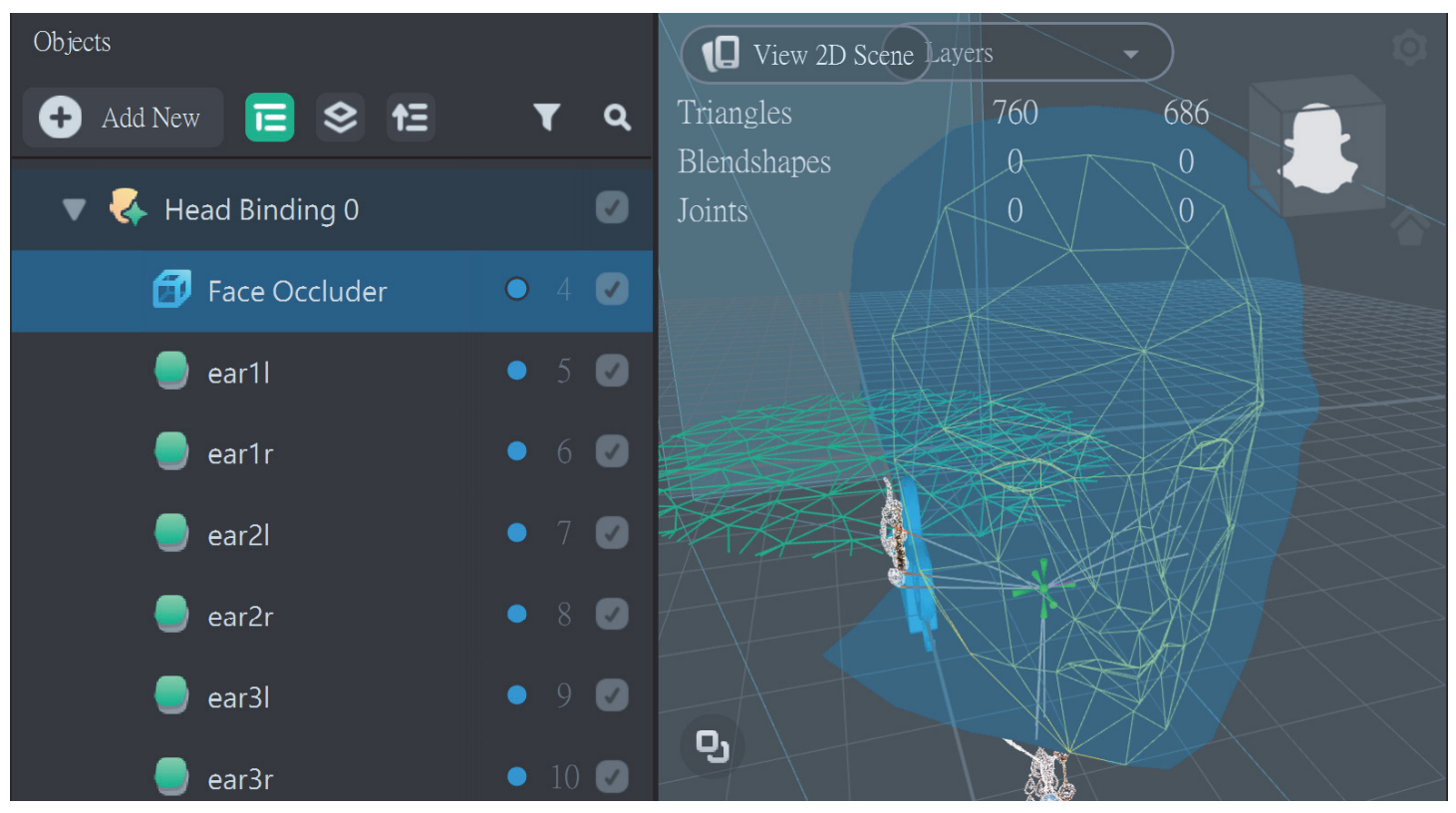


Unlike other virtual jewellery fittings which only overlay jewellery in a static photo, the head binding allows customers to live preview the dynamic effect of the virtual jewellery fitting in real-time without any markers or initial positioning.

First, when a 2D face is identified, a 3D head model will be projected onto the 2D face according to its angles and positions. Second, the neck and ear positions of the 3D head model are determined, and the jewellery products are displayed onto the customer's neck and ears. Third, the occluder occludes the jewellery which is hidden at a certain angle, e.g. the necklace behind the neck or the earring behind the face. Lastly, the virtual jewellery will move and tilt according to the angle and movement of the 3D head model in real time.

\section{Hand Recognition Module}

After preparing the face recognition module, hand recognition is set up in Lens Studio. The hand tracking module tracks the hand position and overlays virtual jewellery onto the customer's hand. The virtual jewellery's rotation and scale are adjusted according to the hand rotation and the distance between the hand and the camera.

\section{Overlay Virtual Jewellery}

After the setup of the face and hand recognition modules, 2D jewellery images can be assigned to the sprite object and overlaid onto the head binding and hand tracking. Sprite object is a tool to display 2D photos and position it in 3D. The sprite object is attached to the centre of the head binding as ears and neck are not facial landmarks in Lens Studio. The 3D position of the sprite object is 1) manually scaled to the correct proportion; 2) moved to the correct position; 3) rotated to the correct angle; and 4) flipped to create the earring pair. The necklace is created following the same process.

3D models in Filmbox (FBX) or Wavefront Object (OBJ) file formats with less than 5,000 polygons are supported by Lens Studio. 3D models can be constructed through Rhino, Matrix, or 3D scanning and rendered through Keyshot. The rendered 3D model is added to the head binding. Rendered finishing is added to the material to display the colour of the 3D model. 
FIGURE 2

SCRIPT EXAMPLE OF SELECTING JEWELLERY

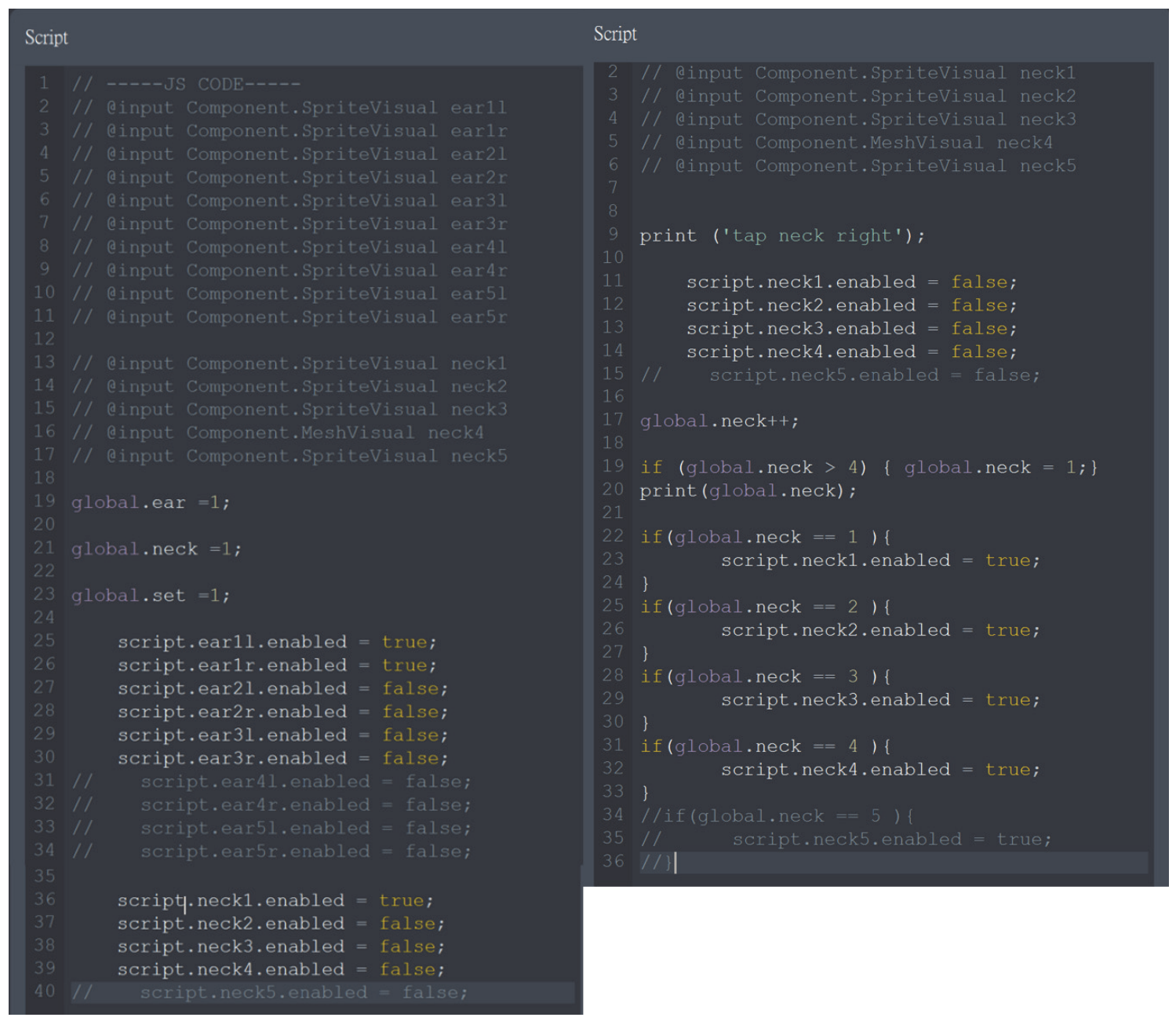

\section{User Interface for Jewellery Selection \\ Button}

After setting up the basic functions, buttons are created to control the MRVJF. Buttons are triggers for customers to select different combinations of earrings and necklaces and perform different virtual fitting features. Transparent buttons are created by adding a sprite object to the orthographic camera and changing the alpha to 0 . In the MRVJF, three rows and three columns of buttons are created. The three rows of buttons control the earring, jewellery set and necklace respectively. The three columns of buttons allow customers to 1) select the previously viewed jewellery; 2) hide the currently selected jewellery; and 3 ) select the next jewellery respectively. A touch component is added for buttons to detect the tapped action. A script component is added to control the assigned actions.

Script

A java scripting library is available for developers to create complex and interactive features. With scripting, developers can create their own physics engines and games. In the MRVJF, an initialised script is run at start-up to display the first set of jewellery. Variables in the script are defined and connected with the objects in the lens. In the left of figure 2, 2D MeshVisual variables are defined in rows 2 to 17 as the examples. Rows 19-23 are examples of defining global variables as the counters to switch jewellery 
image. The display of virtual jewellery is controlled by enabling or disabling the sprite objects in rows 25 40. The right script controls the switching of jewellery by changing the global variable counter.

\section{FIGURE 3 \\ LAYOUT, SETTINGS AND EFFECTS OF THE POSITIONAL FINE-TUNING ALGORITHM}

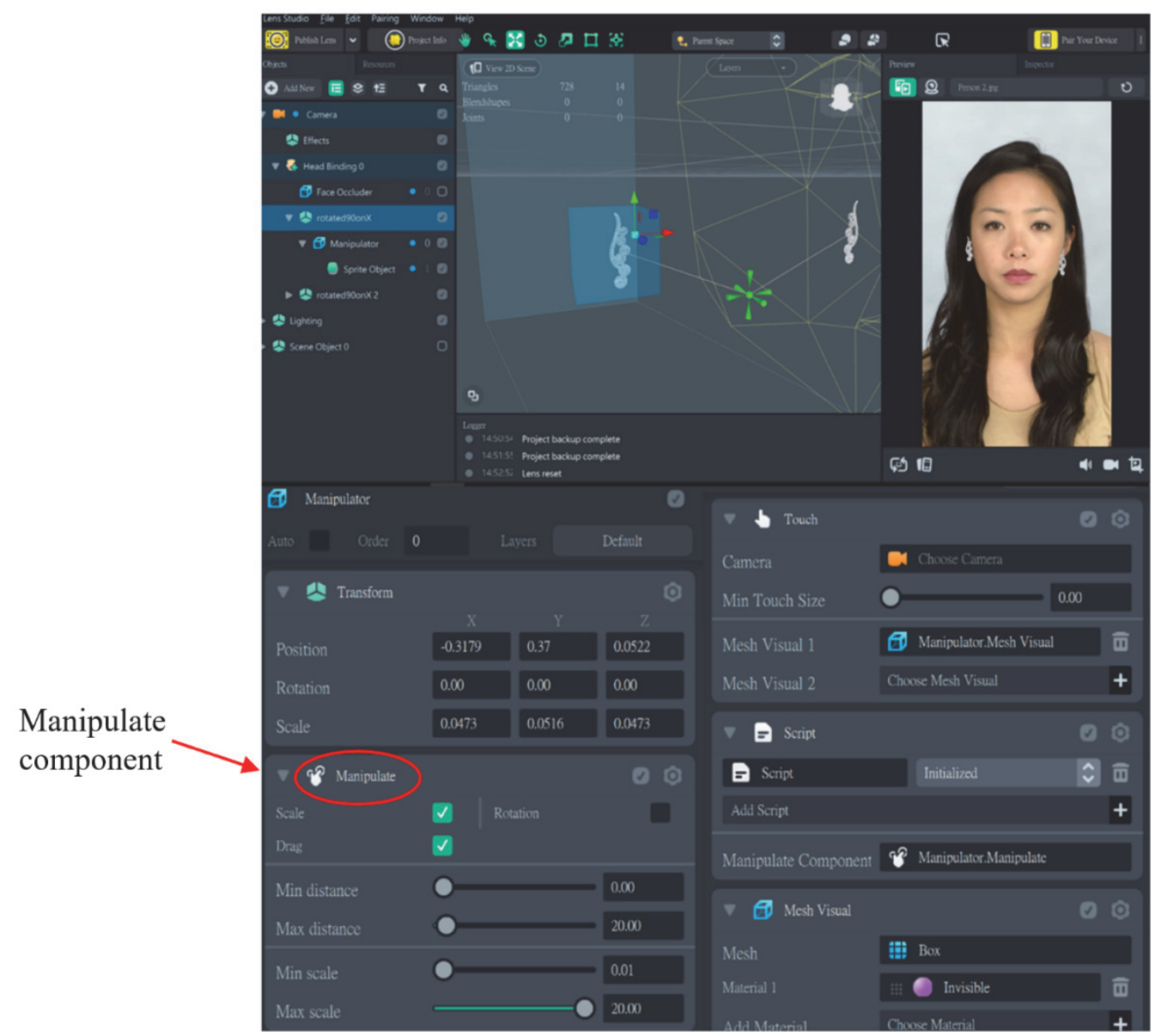

(Team Snap, 2017)

\section{Fine-tuning Jewellery Position}

As ears and neck are not landmarks in face recognition, the position of virtual jewellery may vary on different customers. Furthermore, customers may try on virtual rings on different fingers. Hence, finetuning the jewellery position is required to better fit jewellery onto customers. A dragging algorithm is developed in the MRVJF to allow customers to drag the jewellery into their desired position. The dragging motion does not contradict with the buttons since buttons detect a tap motion and the dragging algorithm detects a tap and hold motion.

Default in Lens Studio, dragging an object up and down will affect its scale as emulating in a 3D space. First, to fix the scale, the object should rotate $90^{\circ}$ along the $\mathrm{x}$-axis. Second, a manipulator box is created to detect the drag command and control the object position. Third, a 'manipulate component' (in figure 3) with 1) deselected rotation; 2) minimum distance being -999; and 3) maximum distance being 20 , is added to the manipulator box. The minimum distance allows the box to pass through the centre position. The maximum distance controls how far the object can be dragged away from the centre position which prevents jewellery from being dragged out of the screen. Fourth, a touch component is added to the manipulator box for detecting the drag command. Depth test, depth write and colour mask are deselected to create an invisible material for the box to avoid covering the jewellery. Fifth, jewellery is added to the 
manipulator box with a $-90^{\circ}$ rotation along the $\mathrm{x}$-axis to counteract with the previous rotation. Lastly, the object, the box, and the jewellery should be fixed in the same position with correct scaling for proper dragging effect. Figure 3 demonstrates the layout, settings, and effects of the positional fine-tuning algorithm.

\section{Beauty Enhancement \\ Face Stretch}

Face stretch transforms the shape of the customer's face into a slimmer and prettier visualisation by modifying the position, size, and the shape of the customer's eyes, nose, and face. In the MRVJF, big eyes, small and tall noses, small mouth, and a slim face are applied.

\section{Face Retouch}

Face retouch covers some minor blemishes on the face, include smoothening the pimples on the skin, softening skin texture, whitening the teeth, and sharpening the eyes. In the MRVJF, the skin texture is softened and the eyes are sharpened.

\section{Face Paint}

Face paint utilises graphics to create genuine makeup effects. By adding graphics to the Photoshop template, eye makeup and face makeup can be created.

\section{Visualisation Improvement}

Guidelines for Jewellery Photography

Jewellery angles and positions affect the authenticity of virtual fitting effects. To simulate the outlook of wearing the earrings, the earrings should be tilted $10^{\circ}$ from the camera during photo shooting. Necklace photography requires a real size necklace stand or a model. By taking a front angle necklace photo with real wearing angle and neck size, the virtual necklace will look more naturally lying on the neck. Ring photography requires a front-facing angle image to prevent capturing the back of the ring band.

A high-resolution camera improves image quality and jewellery attractiveness. Detail background removal is required to create a realistic and beautiful jewellery image. The edge of the necklace is recommended to fade naturally to smoothly fit on customers. The real jewellery and its image should have a 1:1 ratio to provide accurate size information.

\section{Background Segmentation}

Background segmentation improves the immersive experience in virtual fitting. Different backgrounds including garden, church, and performing stage can be applied to visualise jewellery fitting on different occasions. The mechanism of the background segmentation is first by segmenting the outline and facial features of the person from the background. Then, the new background is overlaid behind customers. Two extra cameras named camera masked and orthographic camera masked are created for background segmentation. The camera masked controls the segmentation effect on the face and body. The orthographic camera masked controls the new background. The new background is created by adding a sprite object to the orthographic camera mask. Advanced settings are manipulated in the script of 'Segmentation Controller'. Figure 4 demonstrates the layout and effects of the background segmentation.

Apart from background segmentation, shoulder, face, and sky segmentation are available for dress-up functions or simulating a baby with parent's facial characteristics. 
FIGURE 4

\section{LAYOUT AND EFFECTS OF THE BACKGROUND SEGMENTATION}

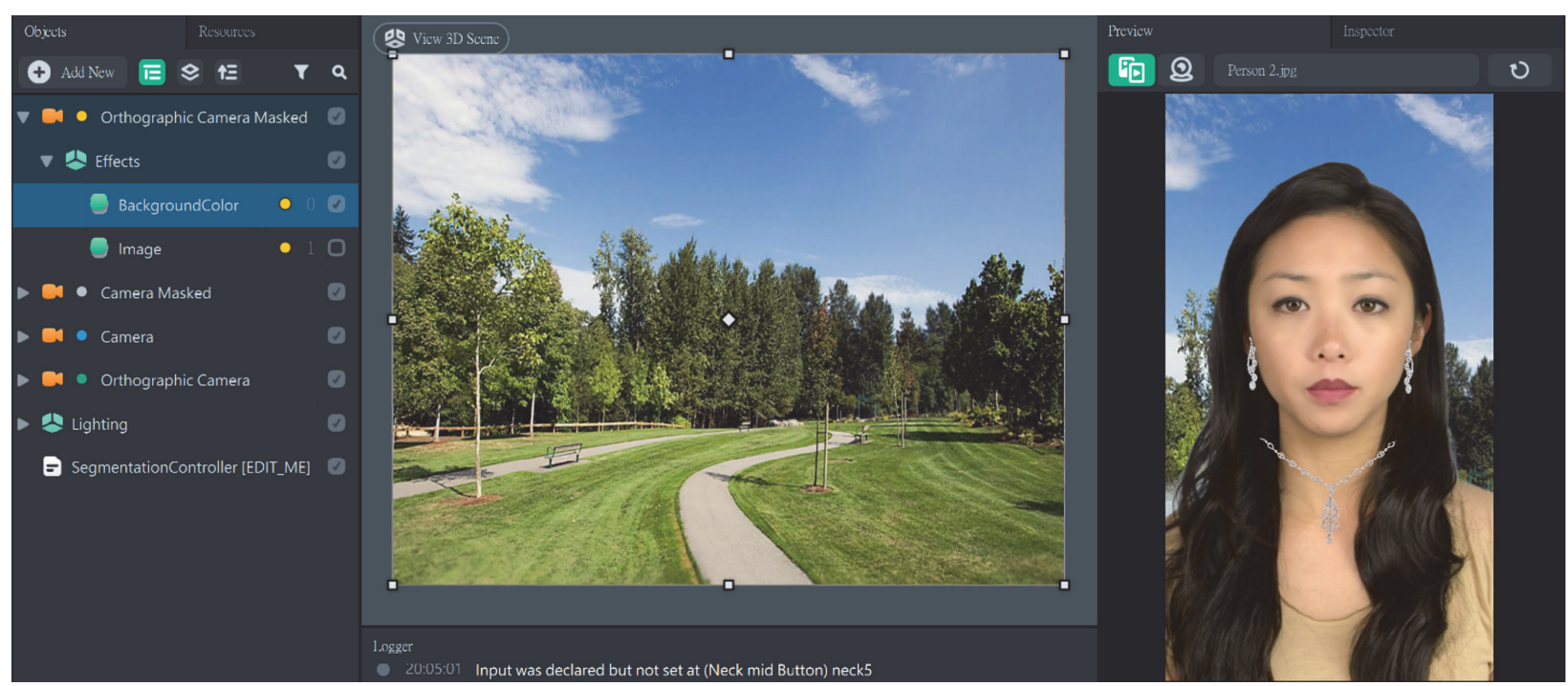

(Team Snap, 2018)

\section{Interactive Multi-person Recognition}

Lens Studio supports multiple faces recognition. By modifying the face index property, jewellery can be overlaid on multiple customers. Multi-person recognition enhances virtual jewellery fitting experiences and interactions.

\section{Gravitational Effect on Virtual Jewellery}

Default in Lens Studio, the overlaid jewellery rotates according to the head rotation, causing virtual jewellery to disobey Newton's law of gravity. To simulate the effect of gravity, additional scripts are implemented to track the head position without capturing the head rotation. With the modification, a jewellery always points downward due to gravity.

\section{Summary}

This paper proposes the development of a real-time interactive mobile MRVJF which integrates face recognition, hand recognition, and mixed reality technologies to improve the shopping experience of customers who can virtually fit jewellery anytime anywhere. The MRVJF can overlay jewellery products onto customers to visualise the fitting effect. To enhance the virtual fitting experience, new features including position fine-tuning, background segmentation, multi-person virtual jewellery fitting, and makeup are developed. A dragging algorithm is developed enabling customers to fine-tune the position of the virtual jewellery. Jewellery photo shooting requirements are proposed to improve virtual jewellery realism.

The major significance of the research is the development and breakthrough in the MRVJF. First, the bottlenecks of the MRVJF including real-time positional tracking, interactive fitting experience, and jewellery realism are tackled. Second, customers can clearly visualise and virtually fit jewellery online instead of a retail store. Third, the MRVJF facilitates pre-purchase services and promotes e-commerce. Fourth, the MRVJF is a unique promotional tool for developing jewellery branding. Last, visualisation realism is improved to make MR for business usage possible.

Compared with existing virtual jewellery fittings, the MRVJF tackles a few limitations, including 1) manual and marker positioning; 2) static jewellery fitting; 3) lack of real-time interaction; 4) artificial visualisation; and 5) lack of mobility. First, real-time face recognition is utilised for positional tracking without the aid of manual or marker positioning. Second, real-time jewellery fitting is available in the 
MRVJF compared with the static image jewellery fitting in other applications. Third, interactive jewellery fitting is available in the MRVJF utilising mixed reality technology. Fourth, the realism of the jewellery model is improved by the proposed photo shooting requirements. Lastly, the use of mobile devices makes the MRVJF convenient for virtual jewellery fitting anytime anywhere.

\section{DISCUSSION}

\section{Comparison with Existing Virtual Jewellery Fitting Systems}

Three virtual jewellery fittings including Woman Jewellery Best Jewellery, CaratLane and the MRVJF are compared in table 1 according to the user-friendliness measured by the time required for jewellery fitting. First, the time for initialisation is recorded to analyse the ease of jewellery fitting for new customers. The MRVJF required the least initialisation time ( 25 seconds), followed by the WJBJ (90 seconds) and lastly the CaratLane (100 seconds). Short initialisation duration reduces the barriers of using virtual jewellery fitting.

Second, the time required to fit the first piece of jewellery is recorded to analyse the jewellery fitting efficiency. The MRVJF required the least fitting time ( 5 seconds), followed by the CaratLane (25 seconds) and lastly the WJBJ (90 seconds). Short jewellery fitting duration increases customer incentive to use virtual jewellery fitting.

Thirdly, the time required on jewellery switching is recorded to analyse the ease of fitting different jewellery products. The MRVJF required the least amount of time on jewellery switching ( 0.5 seconds), followed by the CaratLane (10 seconds) and lastly the WJBJ ( 90 seconds). Short jewellery switching duration smoothens customer jewellery fitting experiences and potentially increases the number of fitted products.

TABLE 1

TIME REQUIRED FOR JEWELLERY FITTING WITH EXISTING APPLICATIONS

\begin{tabular}{|l|c|c|c|}
\hline $\begin{array}{c}\text { Application } \\
\text { Time }\end{array}$ & $\begin{array}{c}\text { Woman Jewellery Best } \\
\text { Jewellery (WJBJ) (sec) }\end{array}$ & CaratLane (sec) & MRVJF (sec) \\
\hline Initialisation & 90 & 100 & 25 \\
\hline Fit first jewellery & 90 & 25 & 5 \\
\hline Jewellery Switching & 90 & 10 & 0.5 \\
\hline
\end{tabular}

\section{Performance Indicators for the MRVJF}

The MRVJF has been launched in Snapchat for seven months and receives over 100,000 customer viewings of products. Such a popularity indicates that customers have great interests in the MRVJF. The MRVJF has been shared for over 3,900 times, indicating the number of promotions in Snapchat. The QR code of the MRVJF has been scanned for over 4,500 times, indicating the number of customers visited the MRVJF after being shared. Overall, the MRVJF has been continuously viewed and attracting new customers.

\section{Limitations}

Hand recognition is added to Lens Studio in April 2019, hence it is not as mature as the face recognition. First, it cannot distinguish between the palm and the dorsum of hand which limits the ability to display the back of the ring. Second, for initial hand detection, only front facing hand with fingers straightened can be detected. After the hand is tracked, different hand gesture can be posed without affecting the tracking. Third, the hand cannot be detected when it overlaps with skin-coloured objects. Last, the tilted degree of the hand cannot be captured. Hence, the virtual ring cannot be tilted to visualise its different angles. 
Partial face features reduce accuracy of face recognition. Specifically, when customers look closely at the jewellery, the camera is unable to capture the whole face features and causes unstable jewellery positioning.

The virtual jewellery appears to be separated from the layer of the face due to the differences in lighting and resolution. Apart from overlaying the virtual jewellery, merging its texture with the face is required to improve the realism effect.

The overlaid necklace positions may not match the targeted positions due to the lack of specific landmarks in the neck position and various length and width of customers' neck. Furthermore, no occluder is available to simulate the effect of hair and clothes covering the necklace. To resolve these issues, an algorithm can be implemented to occlude parts of the necklace which does not lie on the fleshcoloured position.

\section{Future Work}

Customer opinions and recommendations will be analysed to improve the MRVJF. Hand recognition will be improved to track tilt movement and display the full angle of the jewellery. Face recognition will be improved to track the face with partial face features. Realism effect will be increased by adding jewellery sparkling effect, metal reflective effect and merging the virtual jewellery with the face layer. Necklace occluder will be developed to improve necklace fitting experiences.

\section{CONCLUSION}

The MRVJF helps jewellery retailing adapt to the future business environment and provides better customer shopping experience with up-to-date digital technology. Critical implementation issues have been addressed to smoothen the development process. The MRVJF provides customers with a real-time interactive way to visualise and virtually fit jewellery. Instead of travelling a long way to a physical store, customers can browse on their phone at home and virtually fit different jewellery products. This will increase customer faith in and motivation for purchasing jewellery online. Furthermore, the virtual fitting experience improves communication between sellers and customers and reduces the risk of customers being dissatisfied with non-returnable tailor-made jewellery. Overall, digitalisation can improve the leading advantages of the Hong Kong jewellery retailers in the global commodity chain and increase their competitiveness in future challenges.

\section{REFERENCES}

Best Photo Apps. (2018). Woman Jewelry Best Jewellery. Retrieved from https://play.google.com/store/apps/details?id=best.photo.app.womanjewelry

Carat Lane. (2018). CaratLane - A Tanishq Partnership - Buy Jewellery. Retrieved from https://play.google.com/store/apps/details?id=com.caratlane.android

Choi, S. H., Yang, Y. X., Yang, B., \& Cheung, H. H. (2015). Item-level RFID for enhancement of customer shopping experience in apparel retail. Computers in Industry, 71, 10-23. doi:10.1016/j.compind.2015.03.003

Cootes, T. F., Taylor, C. J., Cooper, D. H., \& Graham, J. (1995). Active Shape Models-Their Training and Application. Computer Vision and Image Understanding, 61(1), 38-59. doi:10.1006/cviu.1995.1004

Hong Kong Trade Development Council. (2018). Jewellery Industry in Hong Kong. Retrieved from http://hong-kong-economy-research.hktdc.com/business-news/article/Hong-Kong-IndustryProfiles/Jewellery-Industry-in-Hong-Kong/hkip/en/1/1X000000/1X001DFI.htm

Infotech, D. (2018). Jewellery Photo Editor. Retrieved from https://play.google.com/store/apps/details?id=com.devkrushna.editor.jewellery

Intel. (2016). Redefining retail experiences through virtual reality in-stores with intel realsense $3 D$ technology. Retrieved from 
https:/www.intel.co.uk/content/www/uk/en/retail/solutions/documents/redefining-retailexperiences-with-realsense-technology-study.html?_ga=2.208968848.327370846.15523714712021649996.1552371432

Lee, Y.-H., Kim, C., Kim, Y., \& Whangbo, T. (2015). Facial landmarks detection using improved active shape model on android platform. Multimed Tools Appl, 74(20), 8821-8830. doi:10.1007/s11042013-1565-y

Milborrow, S., \& Nicolls, F. (2008). Locating facial features with an extended active shape model, 5305, 504-513.

Optimumbrew Technology. (2017). Try Indian Jewellery On Your Photos. Retrieved from https://play.google.com/store/apps/details?id=com.optimumbrewlab.tryonstudiojewellery

Radhika, Senthil Kumar, T., Privin Dolleth Reyner, P., Leela, G., Mangayarkarasi, N., Abirami, A., \& Vinayaka, K. (2016). 3D Modelling of a Jewellery and Its Virtual Try-on. In Proceedings of the International Conference on Soft Computing Systems (pp. 157-165).

Rodriguez, M., Ajjan, H., \& Peterson, R. (2014). CRM/Social Media Technology: Impact on Customer Orientation Process and Organizational Sales Performance. Journal of Marketing Development and Competitiveness, 8(1), 85-97.

Setyati, E., Kristian, Y., Pranoto, Y. M., \& Alexandre, D. (2013). Augmented reality 3D eyeglasses frame simulator using active shape model and real time face tracking, pp. 1-5.

Statista. (2018). Number of daily active Snapchat users from 1st quarter 2014 to 3rd quarter 2018 (in millions). Retrieved from https:/www.statista.com/statistics/545967/snapchat-app-dau/

Team Snap. (2017). Introducing Lens Studio. Retrieved from https://www.snap.com/enUS/news/post/introducing-lens-studio/

Team Snap. (2018). Segmentation. Retrieved from https://lensstudio.snapchat.com/templates/face/segmentation/

Yang, Y. I., Yang, C.-K., \& Chu, C.-H. (2014). A virtual try-on system in augmented reality using RGB-D cameras for footwear personalization. Journal of Manufacturing Systems, 33(4), 690-698.

Zhang, Q., Guo, Y., Laffont, P.-Y., Martin, T., \& Gross, M. (2017). A Virtual Try-On System for Prescription Eyeglasses. IEEE computer graphics and applications, 37(4), 84-93. 